tool for clinicians in the health care environment enabling quick identification and assignment of individuals that may have a sleep issue.

\section{P075 DOES TOTAL DAILY SCREEN TIME AFFECT OUR SLEEP QUALITY?}

${ }^{1}$ Oscar Benson Gaunt, 'Madelaine Miller, 'Devaki Nimalan*, 'Anežka Macey-Dare, ${ }^{2}$ Mary Morrell, ${ }^{3}$ Chistopher-James Harvey. ${ }^{1}$ Clinical Research and Innovation Theme, Imperial College School of Medicine, London, UK; ${ }^{2}$ Academic Unit of Sleep and Ventilation, National Heart and Lung Institute, Imperial College London, London, UK; ${ }^{3}$ Nuffield Department of Clinical Neurosciences, University of Oxford, UK

\subsection{6/bmjresp-2019-bssconf.75}

Background The percentage of adults spending $>40 \mathrm{hrs} /$ week online has increased by $14 \%$ in the last decade. ${ }^{1}$ Increased screen time is associated with poor sleep quality, which in turn influences memory and attention. This study tested the hypothesis that increased daily screen time was associated with significantly decreased sleep quality.

Methods Data was collected, with ethical approval, over three mornings (May 2019) from 399 randomly selected members of the public in South Kensington. A questionnaire with 15 questions, including age, gender, total daily screen time, sleep onset latency, and daytime alertness was used. A representative sample size of 369 was calculated, based on the daily footfall of Exhibition Road $(32,422) .16$ responses were excluded due to incomplete questionnaires, sleep disorders and jet lag.

Results Data from participants aged 18-34 was selected for analysis ( $\mathrm{n}=223$, 55.9\% of the total responses). Respondents who answered 'no' to the question 'Have you had enough sleep to feel alert?' had a significantly higher mean total screen time than those who answered 'yes' (figure 1: Mean \pm SEM, alert ('yes'): $10 \pm 0.38$ hours, not alert ('no'): 11.2 \pm 0.45 hours, $p=0.02$ ). There was no significant correlation

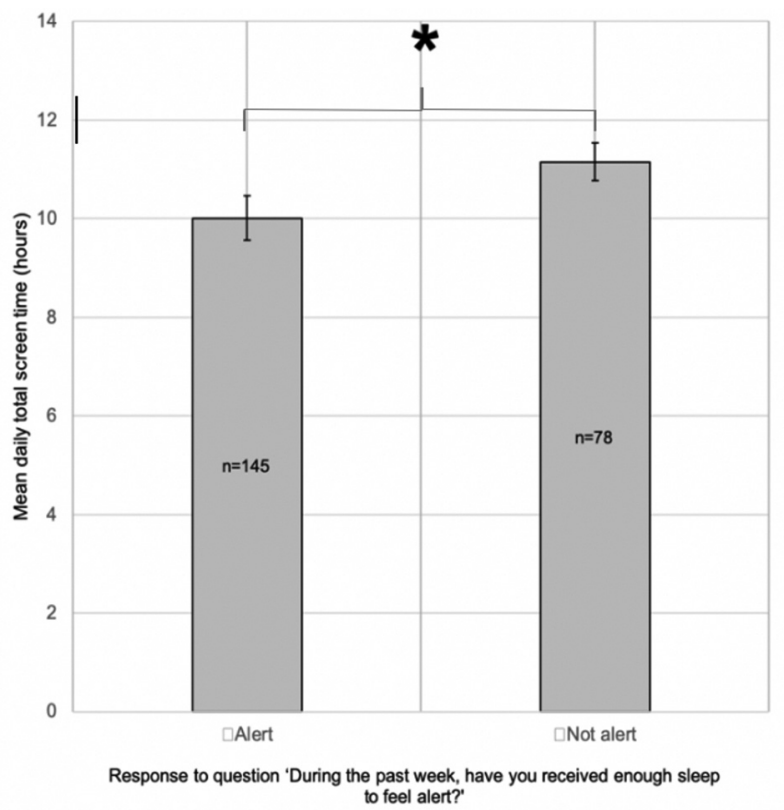

Abstract P075 Figure 1 A bar graph showing self-reported mean daily total screen time (mean \pm SEM) against the responses to the question 'During the pastweek, have you received enough sleep to feel alert?' in 18-34 year olds. There was a significantly higher mean daily screen time in individuals who responded ' $n o$ ' $(p=0.024)$

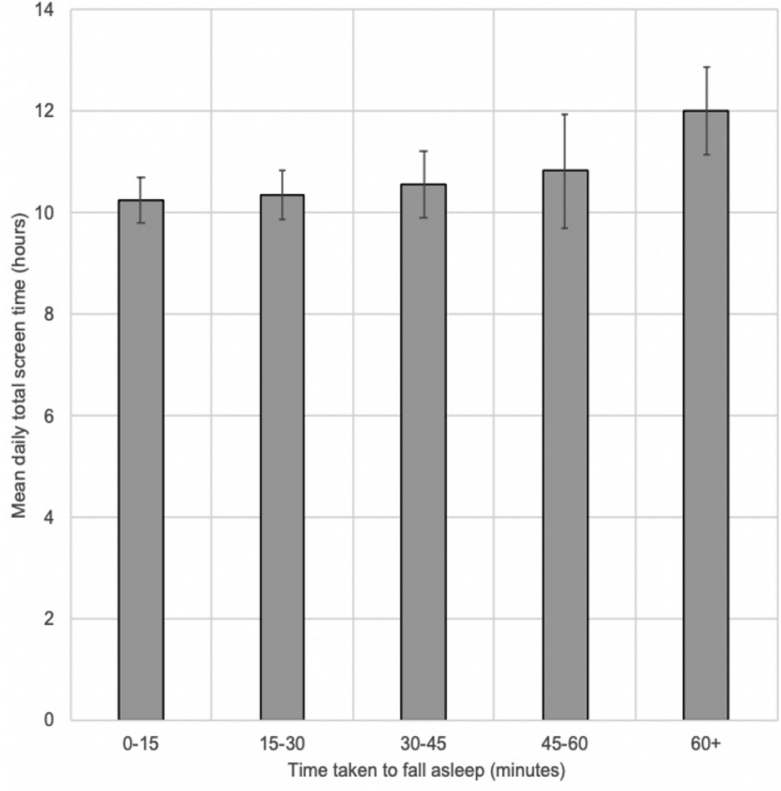

Abstract P075 Figure 2 A bar graph showing self-reported mean dailytotal screen time in hours (mean \pm SEM) against the self-reported sleep onset latency in minutes in 18-34yearolds. No significant correlation was found between daily screen time and sleep onset latency (Spearman's $\rho=0.059$ and $\mathrm{p}=0.38$ )

between the total daily screen time and sleep onset latency (Figure 2: spearman's $\rho=0.059$ and $\mathrm{p}=0.38$ ).

Conclusion The main finding of this study was that increased total daily screen time was associated with reduced daytime alertness, and a reduced sleep quality in members of the public aged 18-34 years. Although, screen time did not have a significant impact on sleep onset latency. This research could potentially raise awareness about the impact of screen time on sleep, and help inform future research into this area.

Acknowledgements With special thanks to our fellow Sleep CRI project members for their contribution to data collection.

\section{REFERENCE}

1. Ofcom. A decade of digital dependency. Available from: https://www.ofcom.org. uk/about-ofcom/latest/features-and-news/decade-of-digital-dependency [Accessed 04.06.19]

\section{P076 SIGNPOSTING FOR SNORING: DOES IT OPTIMISE USE OF GP TIME? ONLINE SURVEYS OF PATIENTS AND SLEEP-TRAINED DENTISTS}

Adrian Zacher* ${ }^{*}$ Emma Easton. Snorer.com, Bicester, UK

\subsection{6/bmiresp-2019-bssconf.76}

Introduction $30_{1}-95_{2} \%$ of adults search online for health information, and online systems are increasingly used to optimise GP clinical time. Intra-oral devices (MADs) are recommended by $\mathrm{NICE}_{3}$ for snoring, mild OSA and where PAP is refused or not complied with.

If SRBD services are to cope with increasing demand, signposting non-somnolent patients (without major co-morbidities) directly to sleep-trained dentists could offer a way to optimise both GP time and improve access to MADs. 